\title{
UNIFORMLY BOUNDED REPRESENTATIONS FOR THE LORENTZ GROUPS
}

\author{
BY \\ EDWARD N. WILSON( $\left.{ }^{1}\right)$
}

\begin{abstract}
A family of uniformly bounded class 1 representations of the Lorentz groups is constructed. This family of representations includes, but is larger than, a similar family of representations constructed by Lipsman. The construction technique relies on a multiplicative analysis of various operators under a Mellin transform.
\end{abstract}

1. Introduction. Let $G=S O_{e}(1, n+1)(n \geqq 2)$ be one of the Lorentz groups. The class 1 principal series of representations of $G$ may be regarded as a family of unitary representations $T(\cdot, s)$ for $s \in i R$. Lipsman constructs a family of representations $R(\cdot, s)$ for $-1<\operatorname{Re} s<1$ with the following properties:

(1) $R(\cdot, s)$ is unitarily equivalent to $T(\cdot, s)$ for $s \in i R$.

(2) $\sup _{g \in G}\|R(g, s)\|<\infty$ for all $s$.

(3) $s \rightarrow R(g, s)$ is an analytic operator-valued function for all $g \in G$.

The main result of this paper is the construction of a family of representations $R(\cdot, s)$ satisfying (1)-(3) for $-n / 2<\operatorname{Re} s<n / 2$.

2. Multiplicative analysis of the Fourier transform on $\boldsymbol{R}^{n}$. Let $n \geqq 2$ be fixed. Denote the standard inner product of vectors $w$ and $z$ in $R^{n}$ by $w \cdot z$ and the length of a vector $w$ by $|w|=(w \cdot w)^{1 / 2}$. Let $S=\left\{w \in \boldsymbol{R}^{n}:|w|=1\right\}$. Identify $\boldsymbol{R}^{n}-\{0\}$ with the Cartesian product $\boldsymbol{R}^{+} \times S$ where $\boldsymbol{R}^{+}$is the multiplicative group of positive real numbers. Denote the usual Lebesgue measures on $R^{n}$ and $S$ by $d w$ and $d \xi$. Define a measure $d^{*} w$ on $\boldsymbol{R}^{n}-\{0\}$ by $d^{*} w=d w /|w|^{n}$. If $d x$ is Lebesgue measure on the real line, then $d x / x$ is a Haar measure for $\boldsymbol{R}^{+}$. Let $L_{2}\left(\boldsymbol{R}^{n}\right), L_{2}\left(\boldsymbol{R}^{n}-\{0\}\right), L_{2}(S)$ and $L_{2}\left(\boldsymbol{R}^{+}\right)$ be the spaces of square integrable functions defined by these measures. By a classical result,

$$
L_{2}(S)=\sum_{k=0}^{\infty} \oplus H_{k}
$$

Received by the editors August 6, 1971.

AMS 1970 subject classifications. Primary 22E43, 22E45; Secondary 42A68, 33A15.

Key words and phrases. Lorentz groups, uniformly bounded representations, multiplicative analysis of Fourier transform, Mellin transform, gamma function, analytic continuation of operators.

(1) The results reported in this paper are part of the author's doctoral dissertation at Washington University. The dissertation was written under the direction of Professor Ray Kunze at the University of California, Irvine. 
where $H_{k}$ is the space of spherical harmonics of order $k$. We may identify $L_{2}\left(R^{n}-\{0\}\right)$ with the Hilbert space tensor product $L_{2}\left(R^{+}\right) \otimes L_{2}(S)$ and hence with

$$
\sum_{k=0}^{\infty} \oplus\left(L_{2}\left(\boldsymbol{R}^{+}\right) \otimes H_{k}\right)
$$

For $f$ a function on $\boldsymbol{R}^{n}$, define $\mathscr{U} f$ on $\boldsymbol{R}^{n}-\{0\}$ by

$$
\mathscr{U} f(w)=|w|^{n / 2} f(w) .
$$

$\mathscr{U}$ is then an isometry of $L_{2}\left(\boldsymbol{R}^{n}\right)$ onto $L_{2}\left(\boldsymbol{R}^{n}-\{0\}\right)$. For

define $\tilde{f}$ on $i R \times S$ by

$$
f \in L_{1}\left(\boldsymbol{R}^{n}-\{0\}\right) \cap L_{2}\left(\boldsymbol{R}^{n}-\{0\}\right)
$$

$$
\tilde{f}(t, \xi)=\int_{0}^{\infty} x^{t} f(x, \xi) \frac{d x}{x}
$$

The mapping $f \rightarrow \tilde{f}$ may be viewed as the tensor product of the identity mapping on $L_{2}(S)$ with the group theoretic Fourier transform mapping integrable functions on $\boldsymbol{R}^{+}$to functions on the dual group $\hat{\boldsymbol{R}}^{+}=i \boldsymbol{R}$. By the Plancherel theorem for locally compact abelian groups, this tensor product of maps has a unique extension to a mapping $\mathscr{M}$ from $L_{2}\left(R^{+}\right) \otimes L_{2}(S)$ onto $L_{2}(i R) \otimes L_{2}(S)$. Up to a scalar multiple, $\mathscr{M}$ is an isometry which will be referred to as the Mellin transform on $L_{2}\left(\boldsymbol{R}^{n}-\{0\}\right)$.

Let $\mathscr{I}$ be the unitary operator on $L_{2}\left(R^{n}-\{0\}\right)$ defined by $(\mathscr{I} f)(x, \xi)=f(1 / x, \xi)$. Let $\mathscr{F}$ denote the Fourier transform on $L_{2}\left(\boldsymbol{R}^{n}\right)$. For $f \in L_{1}\left(\boldsymbol{R}^{n}\right) \cap L_{2}\left(\boldsymbol{R}^{n}\right)$, $\mathscr{F} f$ is defined by

$$
(\mathscr{F} f)(w)=\frac{1}{(2 \pi)^{n / 2}} \int_{R^{n}} f(z) e^{-i w \cdot z} d z
$$

Set $\hat{\mathscr{F}}=\mathscr{I} \mathscr{U} \mathscr{F} \mathscr{U}^{-1}$ and $\tilde{\mathscr{F}}=\mathscr{M} \hat{\mathscr{F}} \mathscr{M}^{-1}$. Then $\hat{\mathscr{F}}$ and $\tilde{\mathscr{F}}$ are unitary operators on $L_{2}\left(R^{+}\right) \otimes L_{2}(S)$ and $L_{2}(i R) \otimes L_{2}(S)$, respectively. The following lemma provides the desired multiplicative analysis of $\mathscr{F}$.

Lemma 1. For $f \in L_{2}(i R) \otimes H_{k}(k=0,1,2, \ldots)$,

$$
\tilde{F} \tilde{f}(t, \xi)=\omega(t, k) \tilde{f}(t, \xi)
$$

where

$$
\omega(t, k)=i^{k} \frac{2^{-t} \Gamma((k+n / 2-t) / 2)}{\Gamma((k+n / 2+t) / 2)}
$$

Proof. Let $\varphi$ be in $H_{k}(k=0,1,2, \ldots)$ and $g$ a $C^{\infty}$ function on $\boldsymbol{R}^{+}$with support in a compact subset of the interval $(0, \infty)$. Since linear combinations of functions of the form $f=g \varphi$ are dense in $L_{2}\left(R^{+}\right) \otimes H_{k}$, it suffices to show that formula (2.5) holds for the function $\tilde{f}=\mathscr{M} f$. Let $\hat{f}=\hat{\mathscr{F}} f$. It follows from Theorem (2.6.1) in Bochner [1, p. 38] that

$$
\hat{f}(x, \xi)=i^{k} g_{1}(x) \varphi(\xi)
$$


where

$$
g_{1}(x)=\int_{0}^{\infty} g(y x) J_{k+(n-2) / 2}(y) d y .
$$

Set $\nu=k+(n-2) / 2$ for convenience in notation. $J_{v}$ is the Bessel function of order $\nu$. Now define

$$
\tilde{g}_{1}(z)=\int_{0}^{\infty} x^{z} g_{1}(x) \frac{d x}{x}=\int_{0}^{\infty} \int_{0}^{\infty} x^{z} g(y x) J_{v}(y) d y \frac{d x}{x}
$$

It can be readily verified that the integral in (2.9) converges absolutely for $\operatorname{Re} z<\nu+1$ and defines an analytic function in this domain. From Titchmarsh $[5$, p. 182],

$$
\int_{0}^{\infty} x^{\alpha} J_{v}(x) \frac{d x}{x}=\frac{2^{\alpha-1} \Gamma((\nu+\alpha) / 2)}{\Gamma((\nu-\alpha+2) / 2)}
$$

for $-\nu<\operatorname{Re} \alpha<\frac{1}{2}$. For $\frac{1}{2}<\operatorname{Re} z<\nu+1$ interchange of the order of integration in (2.9) is valid and yields the result

$$
\tilde{g}_{1}(z)=\frac{2^{-z} \Gamma((\nu+1-z) / 2)}{\Gamma((\nu+1+z) / 2)} \tilde{g}(z)
$$

where

$$
\tilde{g}(z)=\int_{0}^{\infty} x^{z} g(x) \frac{d x}{x}
$$

Since both sides of (2.11) define analytic functions of $z$ in the domain $\operatorname{Re} z<\nu+1$, the result remains valid for $z=t \in i R$. Equation (2.5) now follows from (2.7) and (2.11). This completes the proof of the lemma.

3. Mellin transforms of $A$ and $B$ operators. For $s \in i R$, let $B(s)$ be the unitary operator on $L_{2}\left(R^{n}\right)$ defined by

$$
\begin{aligned}
B(s) f(w) & =|w|^{-s} f(w) & & \text { for } w \neq 0, \\
& =0 & & \text { for } w=0,
\end{aligned}
$$

and set $A(s)=\mathscr{F}^{-1} B(s) \mathscr{F}$.

This section is devoted to establishing a multiplicative analysis of the operators $A(s)$ and $B(s)$. Prior to this, estimates on the growth of the quotient of gamma functions in Lemma 1 must be obtained.

Lemma 2. For $c \in \boldsymbol{R}^{+}$and $z$ in the strip $-c<\operatorname{Re} z<c$, define

$$
F(c, z)=\frac{\Gamma((c+z) / 2)}{\Gamma((c-z) / 2)}
$$

There exists a constant $C$ such that whenever $c>0, \varepsilon>0$ and $z=x+i y$ with $|x|<c-\varepsilon$, then

$$
|F(c, z)| \leqq C e^{3|x|} 2^{-x}(1+2|x| / \varepsilon)|c+2+i y|^{x} .
$$


Proof. The proof is modeled after the argument given by Kunze and Stein $[3$, p. 758] to prove a similar result. From Stirling's formula, for $\alpha>0$ and $w=u$ $+i v \in C$ with $|u|<\alpha$,

$$
\begin{aligned}
\log \frac{\Gamma(\alpha+w)}{\Gamma(\alpha-w)}= & \left(\alpha+w-\frac{1}{2}\right) \log \frac{\alpha+w}{\alpha+i v}-\left(\alpha-w-\frac{1}{2}\right) \log \frac{\alpha-w}{\alpha-i v} \\
& -2 w+i(2 \alpha-1) \arg (\alpha+i v)+2 w \log |\alpha+i v|+B(w)
\end{aligned}
$$

where $B(w)$ is a bounded function. For $\alpha>\frac{1}{2}$ and $|u|<\alpha-\frac{1}{2}$, routine estimates show that

$$
\left|\left(\alpha \pm w-\frac{1}{2}\right) \log ((\alpha \pm w) /(\alpha \pm i v))\right| \leqq 2|u| .
$$

It follows from (3.2) and (3.3) that there exists a constant $C$ independent of $\alpha$ such that

$$
|\Gamma(\alpha+w) / \Gamma(\alpha-w)| \leqq C e^{6|u|}|\alpha+i v|^{2 u} \text { for }|u|<\alpha-\frac{1}{2}
$$

Now let $c>0, \varepsilon>0$ and $z=x+i y$ with $|x|<c-\varepsilon$. Replacing $\alpha$ by $(c+2) / 2$ and $w$ by $z / 2$ in (3.4), we obtain the estimate

$$
\begin{aligned}
|F(c, z)| & =\left|\frac{c-z}{c+z}\right|\left|\frac{\Gamma((c+2+z) / 2)}{\Gamma((c+2-z) / 2)}\right| \\
& \leqq\left|\frac{c-z}{c+z}\right| C e^{3|x|}\left|\frac{c+2+i y}{2}\right|^{x} \leqq C\left(1+\frac{2|x|}{\varepsilon}\right) e^{3|x|} 2^{-x}|c+2+i y|^{x} .
\end{aligned}
$$

LEMMA 3. Let $s=\sigma_{0}+i \tau_{0}$ with $\left|\sigma_{0}\right|<n / 2-\varepsilon$. Set $C_{n}=C^{2} e^{3 n} 2^{n}$. For was in Lemma 1 ,

$$
\sup _{k \in Z^{+} ; t \in i R}|\omega(t+s, k) \omega(t-s, k)| \leqq C_{n}(1+n / \varepsilon)^{2}\left(1+4 / n\left|\tau_{0}\right|\right)^{n / 2}
$$

Proof. From Lemma 2,

$$
\begin{aligned}
\sup _{k \in Z^{+} ; t=i \tau \in i R}|\omega(t+s, k) \omega(t-s, k)| & =\sup _{k, \tau}|F(k+n / 2,-s-t) F(k+n / 2, s-t)| \\
& \leqq C_{n}(1+n / \varepsilon)^{2} \sup _{k, \tau}\left|\frac{k+n / 2+i\left(\tau_{0}-\tau\right)}{k+n / 2-i\left(\tau_{0}+\tau\right)}\right|^{\sigma_{0}} \\
& \leqq C_{n}(1+n / \varepsilon)^{2} \sup _{k, \tau}\left|1+\frac{2\left|\tau_{0}\right|}{|k+n / 2-i \tau|}\right|^{\left|\sigma_{0}\right|} \\
& \leqq C_{n}(1+n / \varepsilon)^{2}\left(1+4 / n\left|\tau_{0}\right|\right)^{n / 2}
\end{aligned}
$$

THEOREM 1. (1) $s \rightarrow A(s) B(s)$ is a commutative family of operators for $s \in i R$.

(2) There exists an operator-valued function $s \rightarrow C(s)$ defined on the strip $-n / 2<\operatorname{Re} s<n / 2$ such that

$$
C(s)=A(s) B(s) A(-s) B(-s) \text { for } \operatorname{Re} s=0 .
$$

(3) $\|C(s)\| \leqq C_{n}(1+n / \varepsilon)^{2}(1+4 / n|\operatorname{Im} s|)^{n / 2}$ for $\varepsilon>0$ and $|\operatorname{Re} s|<n / 2-\varepsilon$.

Proof. Let $\widetilde{B}(s)$ and $\tilde{A}(s)$ be the unitary operators on $L_{2}(i R) \otimes L_{2}(S)$ defined by

$$
\widetilde{B}(s)=(\mathscr{M} \mathscr{U}) B(s)(\mathscr{M} \mathscr{U})^{-1}, \quad \tilde{A}(s)=(\mathscr{M} \mathscr{U}) A(s)(\mathscr{M} \mathscr{U})^{-1} \text {. }
$$


It is easily checked that for $f \in L_{2}(i R) \otimes L_{2}(S)$,

$$
\begin{aligned}
\tilde{B}(s) f(t, \xi) & =f(t-s, \xi), \\
\tilde{A}(s) & =\mathscr{F}^{-1} \widetilde{B}(-s) \tilde{F} .
\end{aligned}
$$

From (3.6), (3.7) and Lemma 1, it follows that for $f \in L_{2}(i R) \otimes H_{k}(k=0,1,2, \ldots)$,

$$
\tilde{A}(s) \widetilde{B}(s) f(t, \xi)=(\omega(t+s, k) / \omega(t, k)) f(t, \xi) .
$$

(1) is an immediate consequence of (3.8). Now for $|\operatorname{Re} s|<n / 2$, define $\widetilde{C}(s)$ on $L_{2}(i R) \otimes H_{k}$ by

$$
\widetilde{C}(s) f(t, \xi)=\frac{\omega(t+s, k)}{\omega(t, k)} \frac{\omega(t-s, k)}{\omega(t, k)} f(t, \xi) .
$$

From Lemma 3, the multiplier in (3.9) is a bounded function of $t \in i \boldsymbol{R}$ and $k \in \boldsymbol{Z}^{+}$ for each $s$ in the strip $|\operatorname{Re} s|<n / 2$. Hence (3.9) defines a bounded operator on $L_{2}(i R) \otimes L_{2}(S)$. Clearly $s \rightarrow \widetilde{C}(s)$ is analytic. Defining $C(s)=(\mathscr{M} \mathscr{U})^{-1} \widetilde{C}(s) \mathscr{M} \mathscr{U}$, we obtain an analytic operator-valued function on $L_{2}\left(R^{n}\right)$ satisfying (2) and (3).

4. Construction of uniformly bounded representations for the Lorentz groups. For $n \geqq 1$, define $G=S O_{e}(1, n+1)$ to be the connected component of the identity in the group of $(n+2) \times(n+2)$ real matrices $g$ for which ${ }^{t} g p_{0} g=p_{0}$ where ${ }^{t} g$ is the transpose of $g$ and

$$
p_{0}=\left[\begin{array}{ccc}
0 & 0 & 1 \\
0 & -I_{n} & 0 \\
1 & 0 & 0
\end{array}\right] .
$$

The family of Lorentz groups is the collection $\left\{S O_{e}(1, n+1): n \geqq 1\right\}$. For $n=1$, the group $S O_{e}(1,2)$ is locally isomorphic to $S L(2, R)$. Uniformly bounded representations of this group are constructed by Kunze and Stein [2]. A modified version of the Mellin transform analysis used here applies to this group but yields no new representations. We shall therefore assume $n \geqq 2$ in the remainder of this paper.

Let $M, A, N$ and $V$ be the subgroups of $G$ defined as follows:

$$
\begin{aligned}
& A=\left\{a=a(x)=\left[\begin{array}{ccc}
x & 0 & 0 \\
0 & I_{n} & 0 \\
0 & 0 & 1 / x
\end{array}\right]: x \in \boldsymbol{R}^{+}\right\}, \\
& M=\left\{m=m(h)=\left[\begin{array}{lll}
1 & 0 & 0 \\
0 & h & 0 \\
0 & 0 & 1
\end{array}\right]: h \in S O(n, \boldsymbol{R})\right\}, \\
& N=\left\{u=u(w)=\left[\begin{array}{ccc}
1 & w & \frac{1}{2}|w|^{2} \\
0 & I_{n} & { }^{t} w \\
0 & 0 & 1
\end{array}\right]: w \in \boldsymbol{R}^{1 \times n}\right\}, \\
& V=\left\{v=v(w)={ }^{t} u(w)\right\} .
\end{aligned}
$$


Set $B=M A N$. For $n$ odd, set $J_{n}=-I_{n}$ and for $n$ even, set

$$
J_{n}=\left[\begin{array}{ccccc}
-1 & & & & \\
& -1 & & & \\
& & \ddots & & \\
& & & -1 & \\
& & & & 1
\end{array}\right] .
$$

Now set

$$
p_{n}=\left[\begin{array}{ccc}
0 & 0 & 1 \\
0 & J_{n} & 0 \\
1 & 0 & 0
\end{array}\right]
$$

Throughout the following discussion, $n$ will be fixed and we write $J$ for $J_{n}$ and $p$ for $p_{n}$. Then $p$ is a representative of the nontrivial element of the Weyl group for the rank 1 semisimple Lie group $G . B$ is a closed subgroup of $G$ and by the Bruhat Lemma, $G=B \cup B p B=B \cup B V p$. Set $\widetilde{B}=p^{-1} B p$. Then $\widetilde{B}=M A V$ and $G=\widetilde{B} \cup \widetilde{B} p \widetilde{B}$. The set $B V$ is a dense set of full measure in $G$.

For $s \in i R$, let $T(\cdot, s)$ be the unitary representation of $G$ induced from the character $m a(x) u \rightarrow x^{s}$ of $B$. The family of representations $T(\cdot, s)$ is the class 1 principal series of $G$. These representations may be realized as operators on $L_{2}(V)$ defined by the formulae

$$
\begin{aligned}
T(g, s) f(v) & =x^{n / 2+s} f(v \cdot g) & & \text { if } v g=(m a(x) u)(v \cdot g) \in B V, \\
& =0 & & \text { if } v g \notin B V .
\end{aligned}
$$

Since $w \rightarrow v(w)$ is a measure preserving homeomorphism of $\boldsymbol{R}^{n}$ onto $V$, we may identify $L_{2}(V)$ with $L_{2}\left(R^{n}\right)$ by writing $f(w)$ for $f(v(w))$. Note that

$$
v(w) a(x)=a(x) v(x w) \text { for all }(x, w) \in \boldsymbol{R}^{+} \times \boldsymbol{R}^{n}
$$

and

$$
v(w) m(h)=m(h) v(w h) \quad \text { for all }(h, w) \in S O(n, R) \times \boldsymbol{R}^{n} .
$$

In (4.3) $w h$ is to be interpreted as the product of the row matrix $w$ and the orthogonal matrix $h$. From (4.1)-(4.3), it follows that the operators $T(g, s)$ for $g=$ $m(h) a(x) v\left(w_{0}\right) \in \widetilde{B}$ are defined on $L_{2}\left(\boldsymbol{R}^{n}\right)$ by

$$
T\left(m(h) a(x) v\left(w_{0}\right), s\right) f(w)=x^{n / 2+s} f\left(x w h+w_{0}\right) .
$$

An easy matrix calculation shows that for $w \neq 0$,

$$
v(w) p=m\left(J-2^{t} w w J /|w|^{2}\right) a\left(2 /|w|^{2}\right) u(-w J) v\left(2 w J /|w|^{2}\right) .
$$

For $f \in L_{2}\left(\boldsymbol{R}^{n}\right)$ it follows that

$$
\begin{aligned}
T(p, s) f(w) & =\left(2 /|w|^{2}\right)^{n / 2+s} f\left(2 w J /|w|^{2}\right) & & \text { for } w \neq 0 \\
& =0 & & \text { if } w=0 .
\end{aligned}
$$


Since $G=\widetilde{B} \cup \widetilde{B} p \widetilde{B}$, the representation $T(\cdot, s)$ is completely determined by (4.4) and (4.5). Now define $R(\cdot, s)=A(s) T(\cdot, s) A(-s)$.

LeMmA 4. $\left.R(\cdot, s)\right|_{\tilde{B}}=\left.T(\cdot, 0)\right|_{\tilde{B}}$.

Proof. Since $A(s)=\mathscr{F}^{-1} B(s) \mathscr{F}$, it suffices to show that

$$
B(s) \hat{T}(g, s)=\hat{T}(g, 0) B(s)
$$

for all $g \in \widetilde{B}$ where $\hat{T}(g, s) \equiv \mathscr{F} T(g, s) \mathscr{F}^{-1}$. Let $g=m(h) a(x) v\left(w_{0}\right)$. It follows easily from (4.4) that

$$
\hat{T}(g, s) f(w)=x^{-n / 2+s} e^{i w_{0} \cdot x^{-1} w h} f\left(x^{-1} w h\right) .
$$

Formula (4.6) is an immediate consequence of (4.7).

Lemma 5. $A(2 s) T(\cdot, s)=T(\cdot,-s) A(2 s)$.

Proof. From (4.7) it follows easily that

$$
B(2 s) \hat{T}(g, s)=\hat{T}(g,-s) B(2 s) \text { for all } g \in \tilde{B} .
$$

Hence it suffices to show that

$$
A(2 s) T(p, s)=T(p,-s) A(2 s) \text {. }
$$

Let $\tilde{T}(p, s)=(\mathscr{M} \mathscr{U}) T(p, s)(\mathscr{M} \mathscr{U})^{-1}$. For $f \in L_{2}(i R) \otimes L_{2}(S)$, it follows from (4.5) that

$$
\tilde{T}(p, s) f(t, \xi)=2^{t-s} f(2 s-t, \xi J) .
$$

Note that if $\xi \rightarrow g(\xi)$ is a function in $H_{k}$, then $\xi \rightarrow g(\xi J)$ is also a function in $H_{k}$. Hence the subspaces $L_{2}(i R) \otimes H_{k}(k=0,1,2, \ldots)$ are invariant under $\tilde{T}(p, s)$. From (2.5), (3.7) and (4.8), it follows that for $f \in L_{2}(i R) \otimes H_{k}$,

$$
\tilde{A}(2 s) \tilde{T}(p, s) f=\tilde{T}(p,-s) \tilde{A}(2 s) f .
$$

Formula (4.8) now follows easily from (4.10).

Lemma 6. (1) $R(\cdot, s)=R(\cdot,-s)$ for all $s \in i R$.

(2) $R(p, s)=A(s) B(s) A(-s) B(-s) T(p, 0)$ for all $s \in i R$.

Proof. (1)

$$
\begin{aligned}
R(\cdot, s) & =A(s) T(\cdot, s) A(-s)=A(-s) A(2 s) T(\cdot, s) A(-2 s) A(s) \\
& =A(-s) T(\cdot,-s) A(s)=R(\cdot,-s) .
\end{aligned}
$$

(2) Formula (4.5) implies that $T(p, s)=2^{s} B(2 s) T(p, 0)$. From Lemma 5 and the fact that $T(p, 0)^{2}$ is the identity, it follows that

$$
T(p, 0) A(\cdot,-s) T(p, 0)=2^{-s} B(-s) A(-s) B(-s) .
$$

Hence

$$
\begin{aligned}
R(p, s) & =2^{s} A(s) B(2 s) T(p, 0) A(-s) T(p, 0)^{2} \\
& =A(s) B(s) A(-s) B(-s) T(p, 0) .
\end{aligned}
$$


THEOREM 2. For each $g \in G$, the function $s \rightarrow R(g, s)$ initially defined for $s \in i R$ has an extension to an analytic function on the strip $D=\{s \in C:|\operatorname{Re} s|<n / 2\}$. The resulting operators $R(g, s)$ have the property that $R(g, s)=R(g,-s)$ for all $g \in G$ and $s \in D$. For all $s \in D, g \rightarrow R(g, s)$ is a uniformly bounded representation of $G$.

Proof. Let $s \in D$. For $g \in \widetilde{B}=M A V$, define $R(g, s)=T(g, 0)$. Define $R(p, s)$ $=C(s) T(p, 0)$ where $C(s)$ is the operator-valued function defined in Theorem 1 . If $g \in G$ is of the form $g=g_{1} p g_{2}$ for $g_{1}$ and $g_{2}$ in $\widetilde{B}$, set $R(g, s)=R\left(g_{1}, s\right) R(p, s)$ $R\left(g_{2}, s\right)$. By the preservation of functional equations under analytic continuation and the fact that $G=\widetilde{B} \cup \widetilde{B} p \widetilde{B}$, it follows that the operators $R(g, s)$ are well defined for all $g \in G$ and $s \in D$ and satisfy the symmetry condition $R(g, s)=R(g,-s)$. Moreover, $g \rightarrow R(g, s)$ is a representation of $G$ for all $s \in D$ and $s \rightarrow R(g, s)$ is analytic for all $g \in G$. Since $\sup _{g \in G}\|R(g, s)\|=\|R(p, s)\|=\|C(s)\|$, the representations $R(\cdot, s)$ are uniformly bounded.

\section{REFERENCES}

1. S. Bochner, Harmonic analysis and the theory of probability, Univ. of California Press, Berkeley, Calif., 1955. MR 17, 273.

2. R. A. Kunze and E. M. Stein, Uniformly bounded representations and harmonic analysis of the $2 \times 2$ real unimodular group, Amer. J. Math. 82 (1960), 1-62. MR 29 \#1287.

3. - Uniformly bounded representations. II. Analytic continuation of the principal series of representations of the $n \times n$ complex unimodular group, Amer. J. Math. 83 (1961), 723-786. MR 29 \#1288.

4. R. L. Lipsman, Uniformly bounded representations of the Lorentz groups, Amer. J. Math. 91 (1969), 938-962. MR 42 \#1946.

5. E. C. Titchmarsh, Introduction to the theory of Fourier integrals, 2nd ed., Clarendon Press, Oxford, 1948.

Washington University, St. Louis, Missouri 63130

Current address: Brandeis University, Waltham, Massachusetts 02154 